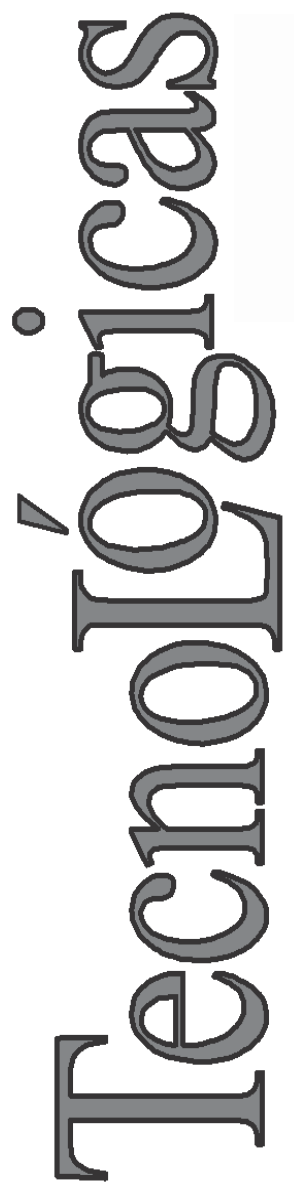

\title{
Modelado y Estimación de Parámetros de un Sistema de Generación Eólico de Baja Potencia
}

\section{Modeling and Parameter Estimation of a Small Wind Generation System}

\author{
Carlos A. Ramírez-Gómez ${ }^{1}$ \\ Andrés J. Saavedra-Montes ${ }^{2}$ \\ Carlos A. Ramos-Paja ${ }^{3}$
}

1 Departamento de Energía Eléctrica y Automática,

Facultad de Minas, Universidad Nacional de Colombia,

Medellín-Colombia

caramireg@unal.edu.co

2 Departamento de Energía Eléctrica y Automática, Facultad de Minas, Universidad Nacional de Colombia, Medellín-Colombia ajsaaved@unal.edu.co

3 Departamento de Energía Eléctrica y Automática, Facultad de Minas, Universidad Nacional de Colombia, Medellín-Colombia caramosp@unal.edu.co 


\title{
Resumen
}

En este artículo se presentan el modelado y la estimación de parámetros de un sistema de generación eólico de baja potencia. El sistema está conformado por: una turbina eólica, un generador de imanes permanentes, un rectificador trifásico y una carga eléctrica de corriente directa. Para estimar los parámetros se utilizan datos de velocidad de viento registrados con una estación meteorológica ubicada en el Campus Fraternidad del ITM. Los datos de velocidad de viento fueron aplicados a un modelo de referencia programado en el software PSIM y de esta simulación se registraron las variables para estimar los parámetros. El modelo del sistema de generación en conjunto con los parámetros estimados constituye una excelente representación del modelo detallado, ofreciendo mayor flexibilidad que el modelo utilizado en el software PSIM.

\section{Palabras clave}

Turbina eólica; generador sincrónico de imanes permanentes; modelo reducido; estimación de parámetros.

\begin{abstract}
The modeling and parameter estimation of a small wind generation system is presented in this paper. The system consists of a wind turbine, a permanent magnet synchronous generator, a three phase rectifier, and a direct current load. In order to estimate the parameters wind speed data are registered in a weather station located in the Fraternidad Campus at ITM. Wind speed data were applied to a reference model programed with PSIM software. From that simulation, variables were registered to estimate the parameters. The wind generation system model together with the estimated parameters is an excellent representation of the detailed model, but the estimated model offers a higher flexibility than the programed model in PSIM software.
\end{abstract}

\section{Keywords}

Wind turbine; permanent magnet synchronous generator; reduced model; parameter estimation. 


\section{INTRODUCCIÓN}

Los sistemas de generación eólica han tenido un gran desarrollo en los últimos años (Barroso, 2010), esto en gran parte por los esfuerzos realizados para disminuir la emisión de gases de invernadero (Moreno, 2010). Los sistemas de generación eólica se convirtieron en una fuente alternativa a las fuentes tradicionales de energía eléctrica, dado que estos sistemas aprovechan la energía disponible en el viento y la transforman en energía eléctrica.

Los sistemas de generación eólica están conformados por una turbina eólica, un generador sincrónico de imanes permanentes (GSIP), un rectificador, un convertidor CD y, dependiendo de la carga, puede incluir o no un inversor (Baroudi, 2007). Para el análisis y control de los sistemas de generación eólica de baja potencia es necesario contar con modelos que simulen su comportamiento.

El modelo del sistema de generación eólico está conformado por un modelo estático que representa la turbina eólica, y un modelo dinámico que representa el acople mecánico entre la turbina y el GSIP. El modelo estático de la turbina eólica posee tres parámetros que son la densidad del aire, el área de barrido y la función del coeficiente de potencia $C p(\lambda)$ (Ramirez \& Saavedra-Montes, 2012). De estos, el más importante es el último ya que define la capacidad de trasformación de energía de la turbina y para encontrarla se han usado diferentes estrategias, las cuales parten con una familia de funciones que tienen un comportamiento similar, pero difieren en el valor de algunas constantes que permiten el ajuste a cada turbina; las funciones usadas son: polinomios de diferentes órdenes (Mok, 2005),(Monroy, 2006), (Sinopoli, 2010), (Son, 2009) y funciones exponenciales (Anaya-Lara, 2009), (Johnson, 1985). En este artículo se presenta una función exponencial $C_{p}$, y un método experimental mediante el cual se encuentra los coeficientes de la función. El método es validado mediante simulación.

Para el modelo dinámico del sistema de generación se diseñó una señal de perturbación basada en un perfil de viento, el cual fue aplicado al sistema de generación programado en software PSIM, de esta se tomaron los datos usados para la estimación de 
parámetros, la cual se realizó herramienta de identificación de parámetros de Matlab y para la validación del modelo obtenido se utilizó el índice de error propuesto por (Penaranda \& SaavedraMontes, 2012).

El presente artículo está conformado de la siguiente forma: la sección 2 se presenta el modelo completo del sistema de generación eólica, en la sección 3 se propone la estimación de parámetros del modelo del sistema de generación y finalmente, en la sección 4 se enuncian unas breves conclusiones sobre el trabajo realizado.

\section{MODELADO DEL SISTEMA DE GENERACIÓN EÓLICO DE BAJA POTENCIA}

El sistema de generación eólico a modelar está conformado por: una turbina eólica, un GSIP, un rectificador trifásico y una carga resistiva. A continuación se describe el modelado de cada uno de los elementos mencionados.

\subsection{Modelo Estático de la Turbina Eólica}

Para modelar la turbina eólica se parte con el cálculo de la potencia disponible en el viento $P_{v}$, la cual se calcula para un área específica $A$, y está dada por (1) (Ramirez \& Saavedra-Montes, 2012).

$P_{v}=0.5 \cdot \rho \cdot A \cdot v^{3}$

Donde la potencia está dada en W, $\rho$ es la densidad del aire en $\mathrm{kg} / \mathrm{m}^{3}, v$ es la velocidad del viento en $\mathrm{m} / \mathrm{s}$ y $A$ está dada en $\mathrm{m}^{2}$. Las turbinas eólicas solo pueden extraer una porción de la potencia presente en el viento; esta porción está representada por el coeficiente de potencia (2),

$C_{p}=P_{t} / P_{v}$

donde $P_{t}$ es la potencia que es aprovechada por la turbina en W. El coeficiente de potencia del rotor de una turbina eólica varía 
en función de la relación de velocidades o $\lambda$, el cual está definido como la relación entre la velocidad de barrido de la turbina y la velocidad del viento. Esta relación se muestra en (3):

$\lambda=\omega \cdot R / v$

El modelo estático de una turbina eólica de tres palas se obtiene a despejar $P_{t}$ de (2) y sustituir $P_{v}$; si además se divide por la velocidad angular, se obtiene el torque mecánico $T_{m}$ en N.m, como se presenta en (4).

$T_{m}=\frac{\rho \cdot A \cdot v^{3} \cdot C_{p}(\lambda)}{\omega}$

Los parámetros que se deben obtener para modelar una turbina eólica son la densidad del aire $\rho$, el área de barrido del rotor $A$ y la función $C_{p}(\lambda)$.

\subsection{Modelo Dinámico de la Turbina y el Generador}

El modelo dinámico de la turbina está dado por la ley de newton para movimiento rotacional, como se muestra en (5) (Mahdi, 2011).

$T_{m}=T_{e}+J \cdot \frac{d \omega}{d t}+F \cdot \omega$

En donde $T_{m}$ y $T_{e}$ son el torque mecánico y el torque eléctrico respectivamente en N.m, $J$ es la inercia mecánica en kg.m² ${ }^{2} \omega$ es la velocidad angular rad/s y $F$ es la fricción del sistema kg.m/s. Si se lleva (5) al dominio de la place y se desprecia el efecto de la fricción se puede encontrar la siguiente función de transferencia que se presenta en (6).

$\frac{\omega}{T_{m}-T_{e}}=\frac{1}{J \cdot s}$ 


\subsection{Modelo del Generador Sincrónico de Imanes Permanentes}

El modelo dinámico del generador sincrónico de imanes permanentes, se puede expresar con la transformación de Park utilizando las ecuaciones (7), (8) y (9) respectivamente. Los subíndices d y q se refieren al eje directo y eje de cuadratura, v es el voltaje, i la corriente, Ls y Rs son la inductancia y la resistencia estator, $p$ es el número de polos y $\mathrm{Apm}$ es el flujo magnético del generador de imanes permanentes (Mahdi 2011).

$v_{d}=R_{s} \cdot i_{d}+L_{s} \cdot \frac{d i_{d}}{d t}-\omega_{e} \cdot L_{s} \cdot i_{q}$

$v_{q}=R_{s} \cdot i_{q}+L_{s} \cdot \frac{d i_{q}}{d t}+\omega_{e} \cdot\left(L_{s} \cdot i_{d}+\lambda_{p m}\right)$

$T_{e}=\frac{3}{2} \cdot p \cdot \lambda_{p m} \cdot i_{q}$

$\mathrm{Al}$ transformar (7) y (8) al dominio de la place, luego multiplicando (8) por $j$ y luego sumarle (7) se obtiene (10). En donde $V_{s}, I_{s}$ son el voltaje y la corriente de fase respectivamente.

$I_{S}(s)=\frac{1}{R_{S}+L_{s} \cdot s} \cdot\left[-V_{s}(s)+p \cdot \lambda_{p m} \cdot(s)\right]$

De la ecuación (10) se puede obtener la función de transferencia que se presenta en (11), en donde $Z_{L}$ es la impedancia equivalente a la salida del generador:

$\frac{I_{s}}{\omega}=\frac{p \cdot \lambda_{p m}}{\left(Z_{L}+R_{S}\right)+L_{s} \cdot S}$

\subsection{Modelo del Rectificador Trifásico}

El rectificador trifásico se modela como una ganancia para el sistema de tal forma que el voltaje y la corriente dc se obtiene con (11) y (12) (Mahdi 2011), en donde $V_{c d}$ y $I_{c d}$ son el voltaje y la corriente a la salida. 


$$
\begin{aligned}
& V_{c d}=\frac{3 \sqrt{6}}{\pi} \cdot V_{s} \\
& I_{c d}=\frac{\pi}{\sqrt{6}} \cdot I_{s}
\end{aligned}
$$

\subsection{Modelo del Sistema de Generación Eólico}

El modelo completo del sistema de generación eólico se presenta en la Fig. 1. En este se muestra el diagrama de bloques y se puede observar la forma en se conectan los modelos de cada uno de los elementos del sistema.

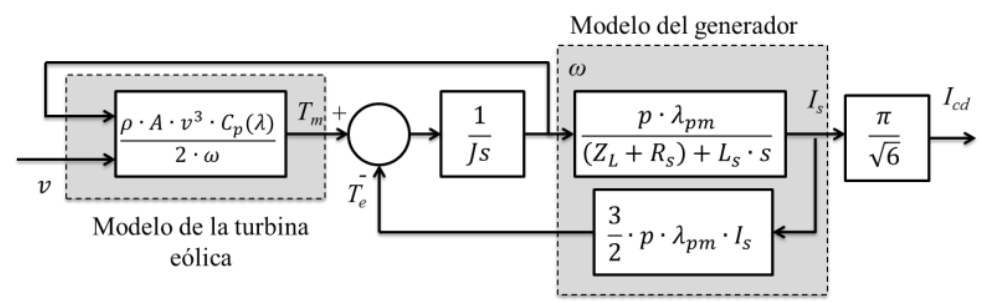

Fig. 1. Diagrama de bloques sistema de generación eólico. Fuente: Autores

\section{IDENTIFICACIÓN DEL SISTEMA DE GENERACIÓN EÓLICO}

Para llevar a cabo la estimación de parámetros del modelo del sistema de generación eólico, se realizará la identificación por separado: en primer lugar se estimarán los parámetros del modelo estático de la turbina eólica, y luego se estimarán los del modelo dinámico del sistema de generación eólico.

\subsection{Identificación del Modelo Estático de la Turbina Eólica}

El modelo estático de la turbina eólica está dado por la ecuación (4); de esta se puede ver que los parámetros a encontrar son $A, \rho$ y la función del coeficiente de potencia $C p$. En el caso de $A$, para una turbina de eje horizontal, se tiene que el área de barrido está dada por la circunferencia formada por las aspas de la turbi- 
na; de esta forma, $A$ se puede calcular con (14), en donde $R$ es el radio de la turbina y es un parámetro de fabricación que se puede obtener de la placa de la turbina eólica.

$A=\pi \cdot R^{2}$

La densidad del aire cambia dependiendo de las condiciones propias de cada lugar; por esta razón debe ser calculada a través de una expresión como la que se enuncia en la ecuación (15), en donde $B$ es la presión atmosférica dada en $\mathrm{kg} / \mathrm{m} . \mathrm{s}^{2}$, $T$ es la temperatura absoluta del aire en $\mathrm{K}$ y $R o$ es la constante del aire seco 287,05 J.kg/K.

$\rho=B /\left(R_{O} T\right)$

Para la construcción de la curva del $C p$, y con el fin garantizar un barrido completo a través de los valores de la curva, es necesario establecer unas condiciones controladas para la prueba. Utilizando el paquete de software PSIM ${ }^{\circledR}$ se simuló la respuesta de una carga mecánica con velocidad angular constante, y como entrada de viento se usó una rampa como la que se muestra en la Fig. 2, en donde también se puede observar la potencia del viento y la potencia mecánica que se entrega a la carga.
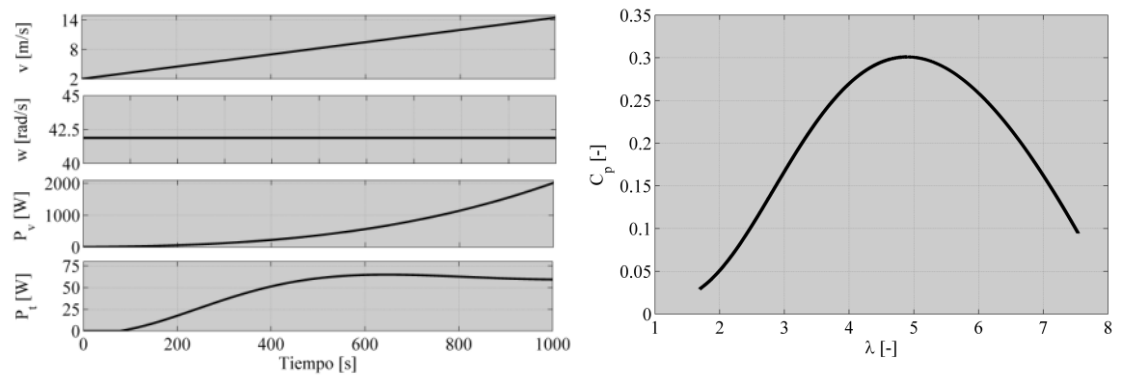

Fig. 2. Identificación del modelo estático de la turbina eólica. Fuente: Autores

En la Fig. 2 se puede ver como al aumentar la velocidad del viento $v$ aumenta la potencia total del viento $P_{v}$, mas la potencia entregada por la turbina eólica $P_{t}$ no lo hace de la misma forma, puesto que llega a un punto máximo y luego desciende levemente. 
Si se grafica la relación de potencias en función de $\lambda$ se obtiene la curva que se presenta a la derecha en la Fig. 2.

Luego de obtener la curva se realiza la aproximación a una función matemática, para realizar esta tarea se utilizó la herramienta "Curve Fitting Tool" del software Matlab®. La función exponencial utilizada para el ajuste está dada en (16), en donde los valores resultantes de los coeficientes fueron: $a=0.3136 ; b=4.944$; $c=2.349$.

$$
C_{p}(\lambda)=a \cdot e^{-((\lambda-b) / c)^{2}}
$$

\subsection{Estimación de Parámetros del Modelo Dinámico del Sistema de Generación}

Para la estimación de los parámetros del modelo dinámico del sistema de generación eólico, se siguieron los siguientes pasos: el primero fue la selección del modelo del sistema de generación eólico; en segundo lugar, se diseñó la señal de perturbación; el tercer paso consistió en la simulación del sistema y recolección de datos; el cuarto paso fue el procesamiento de los datos; luego se realizó la estimación de los parámetros y por último la validación del modelo.

En primer lugar, para modelar el sistema de generación se utiliza el modelo que se presenta en la Fig. 1. Sin embargo, para realizar la identificación del modelo dinámico se tomó el modelo original y se transformó al modelo que se muestra en la Fig. 3, en el cual, la entrada del sistema corresponde al torque mecánico y las salidas son $\omega$ e $I_{c d}$. las ganancias son $k_{1}$ y $k_{2}$, y la constante de tiempo es $\tau_{s}$. Estos últimos tres parámetros están dados por las expresiones (17), (18) y (19).

$$
\begin{aligned}
& k_{1}=\frac{\lambda_{p m} \cdot p \cdot \pi}{\left(Z_{L}+R_{S}\right) \cdot \sqrt{6}} \\
& k_{2}=\frac{3 \cdot \sqrt{6} \cdot \lambda_{p m} \cdot p}{2 \cdot \pi}
\end{aligned}
$$




$$
\tau_{s}=\frac{L_{s}}{Z_{L}+R_{S}}
$$

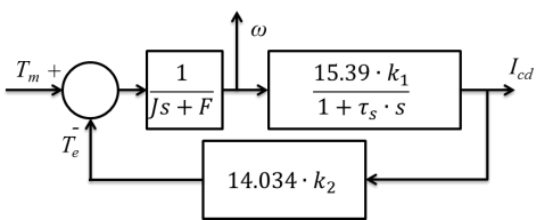

Fig. 3. Diagrama de bloques modelo dinámico del sistema de generación. Fuente: Autores

El segundo paso es el diseño de la señal de perturbación. Para construir la señal, se partió de un perfil de viento tomado en el laboratorio de Energías Alternativas del Instituto Tecnológico Metropolitano de la ciudad de Medellín (ITM). El perfil de viento se modificó con el fin de poder excitar todo el rango de frecuencias de interés (Saavedra-Montes, 2012). En la parte superior de la Fig. 4 se presenta la señal de viento que se usó para excitar al sistema de generación eólico.

En el tercer paso, para la simulación del sistema y la recolección de datos se utilizó el programa PSIM. En la Fig. 4 se muestran las variables medidas: $v, \omega, T_{m}$ e $I_{c d}$. En el paso siguiente, correspondiente al procesamiento de los datos, se realizó un filtrado con el fin de remover el rizado de las señales medidas. En quinto lugar, para la estimación de los parámetros se utilizó la herramienta de estimación de parámetros "Parameter Estimation" de Simulink de Matlab. En la Fig. 5 se observa la comparación de las respuestas del sistema y del modelo estimado para la velocidad angular y la corriente directa; además, los parámetros obtenidos se presentan en la Tabla 1.

Finalmente, para la validación del modelo obtenido se usó el índice Suma Normalizada de los Errores Cuadráticos Modificada SNECM (Penaranda \& Saavedra-Montes, 2012) presentado en la ecuación (20), en donde $y_{i}$ es el dato medido, $\widehat{y}_{i}$ el dato estimado, $\mathrm{y}$ $n$ es el número de datos.

$S N E C M=\sqrt{100^{2} \cdot\left(\frac{\sum_{i=1}^{n}\left(y_{i}-\widehat{y}_{l}\right)^{2}}{\sum_{i=1}^{n}\left(y_{i}\right)^{2}}\right)}$ 
El índice se calculó para cada una de señales de salida del sistema de generación eólica. Para $\omega$ el SNECM es de $0.6696 \%$, y para $I_{c d}$ el SNECM es de $0.5383 \%$.

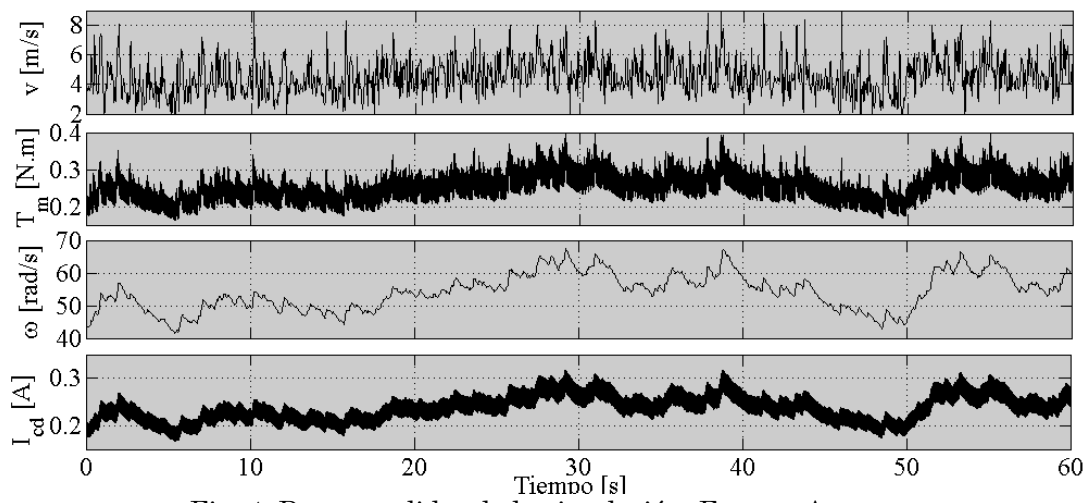

Fig. 4. Datos medidos de la simulación. Fuente: Autores

Tabla 1. Parámetros estimados. Fuente: Autores

\begin{tabular}{cc}
\hline Parámetro & Valor \\
\hline $\mathrm{F}$ & 0.004891 \\
$\mathrm{~J}$ & 0.001024 \\
$\mathrm{~K}_{1}$ & 0.000291 \\
$\mathrm{~K}_{2}$ & $4.554 \mathrm{e}-8$ \\
$\tau_{s}$ & 0.006552 \\
\hline
\end{tabular}
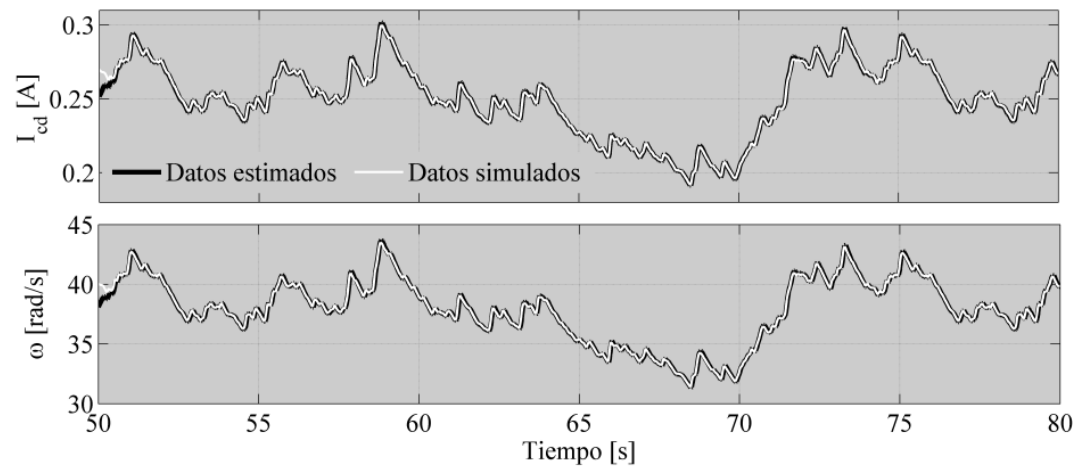

Fig. 5. Datos estimados y datos medidos. Fuentes: Autores 


\section{CONCLUSIONES}

En este artículo fueron presentados el modelo y la estimación de parámetros para un sistema de generación eólico de baja potencia, conformado por una turbina eólica de eje horizontal, un generador sincrónico de imanes permanentes, un rectificador trifásico y la carga eléctrica. Para la estimación de parámetros se tomaron por separado tanto el modelo estático de la turbina eólica como el modelo dinámico del sistema de generación. En el caso del modelo estático, se realizó una construcción de la curva de del coeficiente de potencia $C_{p}$. Para el modelo dinámico del sistema de generación se siguieron cinco pasos, para la estimación de los parámetros se usó la herramienta de Simulink de Matlab y para la validación del modelo obtenido se usó el índice de error MNSSE, para el cual se obtuvo $0.6696 \%$ para la velocidad angular y $0.5383 \%$ para la corriente de carga.

\section{AGRADECIMIENTOS}

Los autores agradecen al programa de jóvenes investigadores 525-2011 y a la beca doctoral 095-2005 de COLCIENCIAS. Este trabajo ha sido soportado por la Universidad Nacional de Colombia bajo los proyectos IDEN-EXGEN y SMART-ALEN.

\section{REFERENCIAS}

Anaya-Lara, O., Jenkins, N., Ekanayake, J., Cartwright, P. \& Hughes, M. (2009). Wind energy generation: modelling and control.).

Baroudi, J. A., Ã, V. D. \& Knight, A. M. (2007). A review of power converter topologies for wind generators. Renewa Energy, 32, 2369-2385.

Barroso, L. A., Rudrick, H., Sensfuss, F. \& Linares, P. (2010). The green effect. Power and Energy Magazine, IEEE, 8(5), 22-35.

Johnson, G. L. (1985). Wind energy systems.).

Mahdi, A. J., Tang, W. H. \& Wu, Q. H. (2011). Derivation of a complete transfer function for a wind turbine generator system by experiments. In Power Engineering and Automation Conference (PEAM), 2011 IEEE,(pp. 35-38). 
Mok, K. (2005). Identification of the Power Coefficient of Wind Turbines. Electrical Engineering), 1-5.

Monroy, A. (2006). Real-time identification of wind turbine rotor power coefficient. Power, 3, 3690-3695.

Moreno, R., Strbac, G., Porrua, F., Mocarquer, S. \& Bezerra, B. (2010). Making room for the boom. Power and Energy Magazine, IEEE, 8(5), 36-46.

Penaranda, J. R. C. \& Saavedra-Montes, A. J. (2012). Dynamic model validation via error indexes. In Circuits and Systems (CWCAS), 2012 IEEE 4th Colombian Workshop on,(pp. 1-6).

Ramirez, C. A. \& Saavedra-Montes, A. J. (2012). Parameter calculation of a small wind turbine model cálculo de parámetros del modelo de una turbina eólica (160 w). In Circuits and Systems (CWCAS), 2012 IEEE 4th Colombian Workshop on,(pp. 1-6).

Saavedra-Montes, A. J., Ramirez-Scarpetta, J. M., Ramos-Paja, C. A. \& Malik, O. P. (2012). Identification of excitation systems with the generator online. Electric Power Systems Research, 87(0), 1-9.

Sinopoli, L. O., M.; Quaicoe, J.E. (2010). Wind turbine rotor modelling using response surface methodology. In Electrical and Computer Engineering (CCECE), 2010 23rd Canadian Conference on,(pp. 1-5).

Son, G., Member, S., Lee, H.-j. \& Park, J.-w. (2009). Estimation of Wind Turbine Rotor Power Coefficient Using RMP Model. Energy). 